\title{
Tuberculosis-HIV co-infection among patients admitted at Muhimbili National Hospital in Dar es salaam, Tanzania
}

\author{
PILI KAMENJU ${ }^{1}$ and SAID ABOUD ${ }^{2 *}$ \\ ${ }^{1}$ School of Medicine, Muhimbili University of Health and Allied Sciences, Dar es Salaam, Tanzania \\ ${ }^{2}$ Department of Microbiology and Immunology, Muhimbili University of Health and Allied Sciences, \\ P.O. Box 65001, Dar es Salaam, Tanzania
}

\begin{abstract}
Data on the clinical presentations and magnitude of tuberculosis (TB)-human immunodeficiency virus (HIV) co-infection is limited. The objective of this study was to describe the clinical presentation and prevalence of TB-HIV co-infection among patients admitted at Muhimbili National Hospital between August 2008 and July 2009 in Dar es Salaam, Tanzania. Of 387 TB patients included, $117(30.2 \%)$ were in the age group 30-39 years. The overall mean age of patients was 38.1 years (range 8 to 86 years) and 216 (55.8\%) were male. Two hundred and five (53\%) had pulmonary tuberculosis (PTB), 58 (15\%) had extra pulmonary tuberculosis (EPTB) and 124 (32\%) had both PTB and EPTB. Common sites of EPTB were pleural cavity $80(44 \%)$, lymph node 31 (17\%) and $30(16.5 \%)$ abdomen. Of the 300 TB patients tested for HIV, 175 (58.3\%) were HIV-infected and $97(55.4 \%)$ of these were already on antiretroviral therapy (ART) at time of admission. Overall, 104 $(26.9 \%)$ of the TB patients admitted died. About two thirds of patients who died had PTB. About three quarters $(73.1 \% ; \mathrm{N}=283)$ of the patients were discharged home to continue with anti-TB treatment. There was significantly higher proportion of deaths among HIV-infected TB patients (29.1\% versus $15.2 \%)$ than in the HIV uninfected TB patients $(P=0.005)$. Age was a significant predictor for PTB (OR 0.946, 95\% CI 0.917-0.976) while age (OR 1.019, 95\% CI 1.002-1.036) and HIV serostatus (OR 2.143, 95\% CI 1.313-3.497) were significant risk factors for EPTB. It is concluded that more than half of the patients had PTB and pleural cavity was the most common site of EPTB. TBHIV co-infected patients had significantly higher mortality than the TB-HIV- uninfected patients. It is recommended to emphasize on the provision of HIV counselling and testing to all TB patients to ensure proper management and timely initiation of ART in TB-HIV co-infected patients in order to improve the outcome and reduce mortality.
\end{abstract}

Key words: Tuberculosis, HIV, extra pulmonary, co-infection, hospital, Tanzania

\section{Introduction}

According to the World Health Organization, an estimated 9.27 million new cases of tuberculosis (TB) occurred in 2007, 31\% in Sub Saharan Africa and 14.8\% of these being among people living with human immunodeficiency virus (PLWHIV) (WHO, 2009). While pulmonary tuberculosis (PTB) is the most common presentation, extra-pulmonary tuberculosis (EPTB) is also an important clinical problem. The term EPTB is used to describe isolated occurrence of TB at body sites other than the lung (Sharma et al., 2004). The case detection rate has been relatively stable since 2001 and well below the global target. The treatment success rate for new smear positive TB cases reached the global target in 2006 (WHO, 2009). Following rapid expansion of collaborative TB-HIV activities, 50\% of TB cases are being tested for HIV and $31 \%$ and $72 \%$ of HIV-infected TB cases are being provided with antiretroviral therapy (ART) and cotrimoxazole prophylaxis (CP), respectively (WHO, 2009).

* Correspondence: Dr. Said Aboud; E-mail: aboudsaid@yahoo.com 
Tanzania ranks $14^{\text {th }}$ among 22 high-burden TB countries in the world (USAID, 2009). The prevalence of TB in Tanzania currently stands at 337 per 100,000 populations. In 2007, an estimated 32,000 Tanzanians died because of TB, $62.5 \%$ of these being PLWHIV. TB has been described as a major public health problem and burden among people living with HIV infection in developing countries (van den Broek et al., 1998; Range et al., 2001; Corbett et al., 2003; Sharma et al., 2005; Somi et al., 2009; WHO, 2009)). In a recent hospital based study in Dar es Salaam, Tanzania, by Bwana et al. (2011) it was reported that over $90 \%$ of the TB patients were also infected with HIV. However, data on the clinical presentations and magnitude of TB-HIV co-infection in Tanzania is limited. The objective of this study was to describe the clinical presentation of TB and determine the prevalence of TB-HIV co-infection among patients admitted at a tertiary hospital in Tanzania.

\section{Materials and Methods}

\section{Study area and subjects}

Retrospective cross-sectional analyses were performed on TB patients admitted in the medical wards at Muhimbili National Hospital (MNH) between August 2008 and July 2009 in Dar es Salaam, Tanzania. MNH is a tertiary referral hospital with a bed capacity of 1400 patients. At $\mathrm{MNH}$, admitted patients diagnosed with $\mathrm{TB}$ are referred to the Directly Observed Treatment Services (DOTS) clinics where they are registered and treated according to the National TB and Leprosy Programme (NTLP). A total of 387 patients' files were retrieved from medical record unit and reviewed. For each patient, data on the sociodemographic, clinical presentation characteristics and diagnosis of TB were recorded. Inclusion criteria included all patients diagnosed with TB (as confirmed by positive acid fast bacilli (AFB) smear results, radiological tests, culture, biopsy or a combination of these).

\section{Diagnosis of tuberculosis}

The clinical case definitions of pulmonary tuberculosis (PTB) and extra-pulmonary TB (EPTB) as recommended by the WHO were used to define the cases included in the study (WHO, 2002). PTB was defined as two or three initial sputum smear examinations positive for AFB, or one sputum smear positive for AFB plus radiographic abnormalities consistent with active pulmonary tuberculosis, as determined by a clinician, or one sputum smear positive for AFB (smear-positive cases). Cases with three sputum smears negative for AFB but clinical and radiological features compatible with active tuberculosis and showing improvement after empirical anti-tuberculosis treatment were considered to be smearnegative cases. Both smear-positive and smear-negative patients were treated as pulmonary TB cases for our data analysis.

EPTB included tuberculosis of organs other than the lungs, such as pleura, lymph nodes, abdomen, genitourinary tract, skin, joints, bones and meninges. Diagnosis of EPTB was based on fine needle aspiration cytology or biochemical analyses of cerebrospinal/pleural/ascitic fluid or histopathological examination or strong clinical evidence consistent with active EPTB, followed by a decision of a clinician to treat with a full course of anti-tuberculosis chemotherapy. Diagnostic procedures included imageological methods, blood tests or laparotomies for excision biopsies. Mantoux tests and BCG challenge tests were also used for the diagnosis of EPTB. In all the cases of EPTB, sputum examinations and chest radiographs were used to investigate the involvement of 
lung parenchyma. Patients diagnosed with TB in both the lungs and an extra-pulmonary site was regarded as having both PTB and EPTB.

\section{Ethical consideration}

Ethical clearance was obtained from the Research and Publication Committee of Muhimbili University of Health and Allied Sciences. Permission to conduct the study and to access the data was obtained from the Muhimbili National Hospital authorities.

\section{Data analysis}

Data were analyzed using the Statistical Package for Social Sciences (SPSS) programme version 15.0. Chi-square $\left(\chi^{2}\right)$ test was used to determine the association between variables. $\mathrm{P}$-value of $<0.05$ was considered significant. Logistic regression analysis was carried out to identify risk factors for being infected with the different clinical forms of TB. Odds ratios (OR) and 95\% confidence intervals (95\% CI) were calculated for each predictor variable.

\section{Results}

Table 1: Baseline socio-demographic and clinical characteristics of the study population $(\mathrm{N}=387)$

\begin{tabular}{|c|c|c|}
\hline Characteristic & Response & Number (\%) \\
\hline \multirow[t]{9}{*}{ Age (years) } & $0-9$ & $2(0.5)$ \\
\hline & $10-19$ & $33(8.5)$ \\
\hline & $20-29$ & $83(21.4)$ \\
\hline & $30-39$ & $117(30.2)$ \\
\hline & $40-49$ & $73(18.9)$ \\
\hline & $50-59$ & $33(8.5)$ \\
\hline & $60-69$ & $19(4.9)$ \\
\hline & $70-79$ & $19(4.9)$ \\
\hline & $80-89$ & $8(2.1)$ \\
\hline \multirow[t]{2}{*}{ Sex } & Male & $216(55.8)$ \\
\hline & Female & $171(44.2)$ \\
\hline \multirow[t]{3}{*}{ History of tuberculosis contact } & Present & $12(3.1)$ \\
\hline & Absent & $11(2.8)$ \\
\hline & Unknown & $364(94.1)$ \\
\hline \multirow{3}{*}{ Clinical presentation } & РTB & $205(53.0)$ \\
\hline & ЕРТВ & $58(15.0)$ \\
\hline & Both PTB and EPTB & $124(32.0)$ \\
\hline \multirow[t]{2}{*}{ HIV status $(n=300)$} & Positive & $175(58.3)$ \\
\hline & Negative & $125(41.7)$ \\
\hline \multirow[t]{2}{*}{ ART use $(n=175)$} & Yes & $78(44.6)$ \\
\hline & No & $97(55.4)$ \\
\hline \multirow[t]{2}{*}{ Patient outcome } & Discharged & $283(73.1)$ \\
\hline & Death & $104(26.9)$ \\
\hline
\end{tabular}

$\mathrm{PTB}=$ pulmonary tuberculosis; $\mathrm{EPTB}=$ extra-pulmonary tuberculosis; $\mathrm{ART}=$ antiretroviral therapy

A total of 387 TB patients were included in the study. The overall mean age of the patients was 38.10 years (range 8 to 86 years). The 30-39 years age group accounted for about onethird $(30.2 \%)$ of the patients (Table 1$)$. Two hundred and sixteen $(55.8 \%)$ were males and $171(44.2 \%)$ were females. The majority of patients $(94.1 \%)$ had unknown history of past contact with an active TB case. One hundred and seventy five (58.3\%) of the $300 \mathrm{~TB}$ 
patients tested for HIV were HIV-infected. Ninety seven (55.4\%) of the HIV infected TB patients were already on antiretroviral therapy (ART) at the time of admission. One hundred and four (26.9\%) of the TB patients admitted in the medical wards died. Two hundred and eighty three $(73.1 \%)$ patients were discharged home to continue with anti-TB treatment (Table 1).

Table 2: Prevalence of PTB and EPTB in relation to socio-demographic and clinical characteristics of the study population $(\mathrm{N}=387)$

\begin{tabular}{lllll}
\hline Characteristics & Response & $\begin{array}{l}\text { PTB (n=205) } \\
\mathbf{n}(\mathbf{\%})\end{array}$ & $\begin{array}{l}\text { EPTB (n=58) } \\
\mathbf{n}(\mathbf{\%})\end{array}$ & $\begin{array}{l}\text { PTB+EPTB (n=12 } \\
\mathbf{n}(\mathbf{\%})\end{array}$ \\
\hline Age (yrs) & $0-9$ & - & $2(100)$ & - \\
& $10-19$ & $11(33.3)$ & $12(36.4)$ & $10(30.3)$ \\
& $20-29$ & $37(44.6)$ & $14(16.9)$ & $32(38.6)$ \\
& $30-39$ & $65(55.6)$ & $19(16.2)$ & $33(28.2)$ \\
& $40-49$ & $39(53.4)$ & $7(9.6)$ & $27(37)$ \\
& $50-59$ & $27(81.8)$ & - & $6(18.2)$ \\
Sex & $60-69$ & $12(63.2)$ & $2(10.5)$ & $5(26.3)$ \\
& $70-79$ & $12(63.2)$ & $1(5.3)$ & $6(31.6)$ \\
History of TB contact & $80-89$ & $2(25)$ & $1(12.5)$ & $5(62.5)$ \\
& $P$-value & 0.001 & $<0.001$ & 0.592 \\
& Male & $124(57.4)$ & $27(12.5)$ & $65(30.1)$ \\
& Female & $81(47.4)$ & $31(18.1)$ & $59(34.5)$ \\
& $P$-value & 0.005 & 0.234 & 0.356 \\
Present & $5(2.4)$ & $3(5.2)$ & $4(3.2)$ \\
HIV status (n=300) & Absent & $7(3.4)$ & $0(0)$ & $4(3.2)$ \\
& Unknown & $193(94.1)$ & $55(94.8)$ & $116(93.5)$ \\
& $P$-value & 0.572 & 0.236 & 0.947 \\
ART use (n=175) & Positive & $103(58.9)$ & $19(10.9)$ & $53(30.3)$ \\
& Negative & $52(41.6)$ & $21(16.8)$ & $52(41.6)$ \\
& $P$-value & 0.003 & 0.135 & 0.043 \\
& Yes & $48(46.6)$ & $7(36.8)$ & $23(43.4)$ \\
& No & $55(53.4)$ & $12(63.2)$ & $30(56.6)$ \\
& $P$-value & 0.518 & 0.473 & 0.837 \\
& Discharged & $142(50.2)$ & $45(15.9)$ & $96(33.9)$ \\
& Death & $63(60.6)$ & $13(12.5)$ & $28(26.9)$ \\
& $P$-value & 0.069 & 0.406 & 0.191 \\
\hline
\end{tabular}

$\mathrm{PTB}=$ pulmonary tuberculosis; $\mathrm{EPTB}=$ extra-pulmonary tuberculosis; $\mathrm{ART}=$ antiretroviral therapy

Two hundred and five (53\%) had PTB, 58 (15\%) had EPTB and 124 (32\%) had both PTB and EPTB (Table 2). Common sites of EPTB ( $n=182)$ were pleural cavity $80(44 \%)$, lymph node $31(17 \%)$ and $30(16.5 \%)$ abdomen, $15(8.2 \%)$ pericardium, $8(4.9 \%)$ pericardium and pleural cavity, 7 (3.8) spine, 3 (1.6\%) abdomen and pericardium, 3 (1.6\%) lymph node and pleural cavity,1 (0.5\%) meninges, 1 (0.5\%) breast, 1 (0.5\%) pleural cavity and abdomen, and 1 $(0.5 \%)$ lymph node, pleural cavity and abdomen. There were statistically significant differences in prevalence of PTB $(P=0.001)$ and EPTB $(P<0.001)$ by age groups. There was significantly higher proportion of PTB cases in male compared to female patients $(P=0.005)$. There were significantly higher proportions $(58.9 \%$ vs. $30.3 \%)$ of PTB $(P=0.003)$ and both PTB \& EPTB ( $p=0.043)$ in HIV-infected compared to HIV-uninfected patients. 
In multivariate analyses, age was a significant predictor for PTB $(0.946,95 \%$ CI $0.917-0.976)$ while age $(1.019,95 \%$ CI 1.002-1.036) and HIV serostatus (2.143, 95\% CI 1.3133.497) were significant predictors for EPTB (Table 3).

Table 3: Predictors associated with TB in the study population $(\mathrm{N}=387)$

\begin{tabular}{lclll}
\hline Risk factor & $\mathbf{n}$ & $\begin{array}{l}\text { PTB } \\
\text { AOR (95\% CI) }\end{array}$ & $\begin{array}{l}\text { EPTB } \\
\text { AOR (95\% CI) }\end{array}$ & $\begin{array}{l}\text { Both PTB \& EPTB } \\
\text { AOR (95\% CI) }\end{array}$ \\
\hline Age: 30-39 years & 117 & $0.946(0.917-0.976)$ & $1.019(1.002-1.036)$ & $1.004(0.987-1.021)$ \\
$\begin{array}{l}\text { HIV status: positive } \\
\begin{array}{l}\text { Patient outcome: } \\
\text { discharged }\end{array}\end{array}$ & 175 & $0.604(0.298-1.222)$ & $2.143(1.313-3.497)$ & $0.629(0.384-1.031)$ \\
\hline
\end{tabular}

\section{Discussion}

Our study findings revealed that the majority of the TB patients at $\mathrm{MNH}$ were in the 30-39 years age group. Our findings differ from previous studies in Nigeria (Ige et al, 2005) and Turkey (Ilgazli et al., 2004) where relatively young individuals were affected. Similar to our findings, the prevalence of TB was reported to be higher in a previous study in Nigeria (Ige et al, 2005). Male sex was significantly associated with PTB. The low proportion of the TB patients who reported to have had past contacts with an active TB case is likely to be due to poor documentation at the hospital. In a recent study in Turkey, Ilgazli et al. (2004) reported that $38 \%$ of the TB patients had history of positive contact with TB cases.

Our study findings revealed that PTB was the most common clinical form of TB at $\mathrm{MNH}$. These findings are similar to those of studies done elsewhere (Ige et al., 2005; Wiwatworapan et al., 2008). TB is transmitted by airborne droplet nuclei, and hence, expose to the infection occurs by sharing common airspace with an individual who is in the infectious stage of TB (Herchline et al., 2009).

The most common sites of EPTB occurrence were the pleura. These study findings are consistent with previous findings in Hong Kong, Madagascar and Turkey (Noertjojo et al., 2002; Rasolofo et al., 2002; Ozvaran et al, 2007). Our findings differed from those of a study conducted in Nepal which revealed that the most common sites of occurrence of EPTB were the lymph node (Sreeramareddy et al., 2008). In the Nepalese study, patients with pleural TB were classified as PTB patients. This could explain the differences in the findings between the two sites.

In our study, over half of the TB patients were also infected with HIV. The prevalence of TB-HIV co-infection was higher that findings reported in Nigeria (Ige et al., 2005; Salami et al., 2006). The reason for the low prevalence of HIV among the TB patients in the two Nigerian studies compared to our study could be due to the fact that the studies were conducted between 1998 and 2004 at a time when there was less emphasis on testing TB patients for HIV as there is now. According to the USAID, collaborative TB-HIV/AIDS services have been scaled up leading to a recent increase in the number of TB patients tested for HIV in Nigeria (USAID, 2009). Of the TB-HIV co-infected patients, many had PTB. Our findings are similar to those of Salami et al. (2006) in Nigeria.

Of the TB-HIV co-infected patients, over half were not on ART at the time of admission at the hospital. A possible reason for this could be that these patients did not 
meet the eligibility criterion used to initiate ART. Another reason could be that some patients might have been eligible to start ART but had not been attending care and treatment clinic for follow up of their health. Slightly more than a quarter of the patients died in the medical wards. This could be due to the fact that many patients sought treatment in the hospital while they had advanced TB disease stage. About two-thirds of the patients who died in the medical wards were PTB patients, probably because overall PTB cases accounted for many of the TB cases. Our study findings suggest that suffering from PTB or EPTB or both PTB and EPTB do not significantly affect patients outcome. However, HIV-infected patients had poor outcome compared to the HIV-uninfected patients.

One study limitation is the fact that this was a retrospective analysis of the TB-HIVco-infected patients and the findings might not be generalizable. The history of past TB contact was not documented in the majority of the patients hence we could not determine the proportion of patients with previous contact who had an active TB case and the association between the past TB contact and the clinical form of TB.

It is concluded that more than half of the patients had PTB and pleural cavity was the most common site of EPTB. It is recommended to emphasize on the provision of HIV counselling and testing to all $\mathrm{TB}$ patients to ensure proper management and timely initiation of ART in TB-HIV co-infected patients in order to improve the outcome and reduce mortality.

\section{Acknowledgements}

We would like to extend our sincere thanks to Mr. Godfrey Sem and the entire team in the medical records unit at $\mathrm{MNH}$ for their invaluable contribution to this report.

\section{References}

Bwana, V.M., Tenu, F., Magesa, S.M. \& Mfinanga, S.G. (2011) Smear positive pulmonary tuberculosis among HIV patients receiving Highly Active Antiretroviral Therapy in Dar es Salaam, Tanzania. Tanzania Journal of Health Research 13, 17-24.

Corbett, E.L., Watt, C.J., Walker, N., Maher, D., Williams, B.G., Raviglione, M.C. \& Dye, C. (2003) The growing burden of tuberculosis global trends and interactions with the HIV epidemic. Archives of Internal Medicine 63, 1009-1021.

Herchline, T. \& Amorosa, J.K. (2009) Tuberculosis, e-medicine. Available at www.emedicine/tuberculosis

Ige, O.M., Sogaolu, O.M. \& Ogunlade, O.A. (2005) Pattern of presentation of tuberculosis and the hospital prevalence of tuberculosis and HIV co-infection in University College Hospital, Ibadan: a review of five years (1998-2002). African Journal of Medicine and Medical Sciences 34, 329-333.

Ilgazli, A., Boyaci, H., Basyigit, I. \& Yildiz, F. (2004) Extrapulmonary tuberculosis: clinical and epidemiologic spectrum of 636 cases. Archives of Medical Research 35, 435-441

Noertjojo, K., Tam, C.M., Chan, S.L. \& Chan-Yeung, M.M. (2002) Extra-pulmonary and pulmonary tuberculosis in Hong Kong. International Journal of Tuberculosis and Lung Disease 6, 879-886.

Ozvaran, M.K., Baran, R., Tor, M., Dilek, I., Demiryontar, D., Arinc, S., Toker, N., Chousein, E.U. \& Sogukpinar, O. (2007) Extrapulmonary tuberculosis in non-human 
immunodeficiency virus-infected adults in an endemic region. Annals of Thoracic Medicine 2, 118-121.

Range, N., Ipuge, Y.A., O’Brien, R.J., Egwaga, S.M., Mfinanga, S.G., Chonde, T.M., Mukadi, Y.D. \& Borgdorff, W.M. (2001) Trend in HIV prevalence among tuberculosis patients in Tanzania, 1991-1998. International Journal of Tuberculosis Lung Disease 5, 405-412.

Rasolofo, R.V., Menard, D., Auregan, G., Gicquel, B. \& Chanteau, S. (2002) Extrapulmonary and pulmonary tuberculosis in Antananarivo (Madagascar): high clustering rate in female patients. J Clinical Microbiology 40, 3964-3969.

Salami, A.K. \& Katibi, I.A. (2006) Human Immunodeficiency virus-associated tuberculosis: pattern and trend in the University of Ilorin Teaching Hospital. African Journal of Medicine and Medical Sciences 35, 457-460.

Sharma, S.K. \& Mohan, A. (2004) Extra pulmonary tuberculosis. Indian Journal of Medical Research 120, 316-353.

Sharma, S.K., Mohan, A. \& Kadhiravan, T. (2005) HIV-TB co-infection: Epidemiology, diagnosis and treatment. Indian Journal of Medical Research 121, 550-567.

Somi, G., Matee, M., Makene, C.L., van den Hombergh, J., Kilama, B., Yahya-Malima, K.I., Masako, P., Sando, D., Ndayongeje, J., Rabiel, B. \& Swai, R.O. (2009) Three years of HIV/AIDS care and treatment services in Tanzania: achievements and challenges. Tanzania Journal of Health Research 11, 136-143.

Sreeramareddy, C.T., Panduru, K.V., Verma, S.C., Joshi, H.S. \& Bates, M.N. (2008) Comparison of pulmonary and extra-pulmonary tuberculosis in Nepal- a hospital based retrospective study. BMC Infectious Diseases 8, 8 .

USAID (2009) Tuberculosis Nigeria Country Profile. Available at http://www.usaid.gov/our work/global health/id/tuberculosis/countries/africa/nig eria_profile.html

van den Broek, J., Mfinanga, S., Moshiro, C., O’Brien, R., Mugomela, A. \& Lefi, M. (1998) Impact of human immunodeficiency virus infection on the outcome of treatment and survival of tuberculosis patients in Mwanza, Tanzania. International Journal of Tuberculosis Lung Disease 2, 547-552

WHO (2002) Global tuberculosis control - surveillance, planning, financing. $\mathrm{WHO} / \mathrm{CDS} / \mathrm{TB} / 2002.295$. Available at http://www.who.int/tb/publications/global report/2002/en/index.html

WHO (2009) Global tuberculosis control-epidemiology, strategy, financing. $\mathrm{WHO} / \mathrm{HTM} / \mathrm{TB} / 2009.411 . \quad$ Available at http://www.who.int/tb/publications/global report/2009/pdf/full report.pdf

Wiwatworapan, T. \& Anantasetagoon, T. (2008) Extra-pulmonary tuberculosis at a regional hospital in Thailand. Southeast Asian Journal of Tropical Medicine and Public Health 39, 521-525 